MATEC Web of Conferences 32, 06005 (2015)

DOI: $10.1051 /$ matecconf $/ 20153206005$

(C) Owned by the authors, published by EDP Sciences, 2015

\title{
Speckle reduction for a laser light sectioning sensor
}

\author{
Rainer Tutsch ${ }^{1, a}$, Sida $\operatorname{Han}^{1}$ and Hanno Dierke ${ }^{1}$ \\ ${ }^{1}$ Institute of Production Metrology, Braunschweig University, Schleinitzstr. 20, 38106 Braunschweig, Germany
}

\begin{abstract}
Automated optical inspection is an important test procedure in electronic circuits assembly. Frequently $3 \mathrm{~d}$ information is required and laser light sectioning sensors are often applied. However, some effects complicate the reliable automatic detection of the shape of such assemblies and their components. The packages of electronic components often are made of black plastics or ceramics so that the intensity available for the optical detection is quite low, especially in comparison to the surface of the PCBs where the components are mounted on. In addition due to rough surfaces of the components and the coherence length of the laser light speckle structures arise. In the work presented here a piezo actuator is used to oscillate the illuminating laser lines along the direction of the lines. The aim is to reduce the visibility of the speckle structures by averaging while maintaining the geometrical shape of the lines. In addition, image processing methods like segmentation and skeletonization are used to allow the detection of the shape of components and assemblies also if materials with distinct differences in the reflectivity are involved. Investigations include the influence of the parameters amplitude and frequency of the piezo actuator.
\end{abstract}

\section{Introduction}

Automated Optical Inspection (AOI) is an important technique in quality assurance in electronic circuits assembly. Electronic cameras and digital image processing are applied to check the completeness of assembly and to verify the correct type, position and orientation of the components on the board. In recent years the evaluation of the $3 \mathrm{~d}$ information came into focus. Measurement of the height profile of electronic components can be applied to detect certain characteristic errors like tombstoning of small components. Furthermore information about the quality of the solder joints of Ball Grid Array (BGA) components can be derived from the height profile.

Electronic cameras give images with $2 \mathrm{~d}$ information. One way to enhance this to the third dimension is the application of structured illumination. As an example fig. 1 shows the basic setup of a laser light sectioning sensor. The working principle can be traced back to a technique invented for microscopy by G. Schmaltz about 80 years ago [1]. A laser beam is expanded in one direction, e.g. using a cylinder lens. The laser line is projected onto the workpiece and an image is taken by a camera with an inclination angle between the optical axis of the imaging lens and the projection direction of the laser line. The height profile of the surface of the workpiece can then be calculated from the local shift of the laser line in the image.

\footnotetext{
${ }^{\mathrm{a}}$ Corresponding author: r.tutsch@tu-bs.de
}

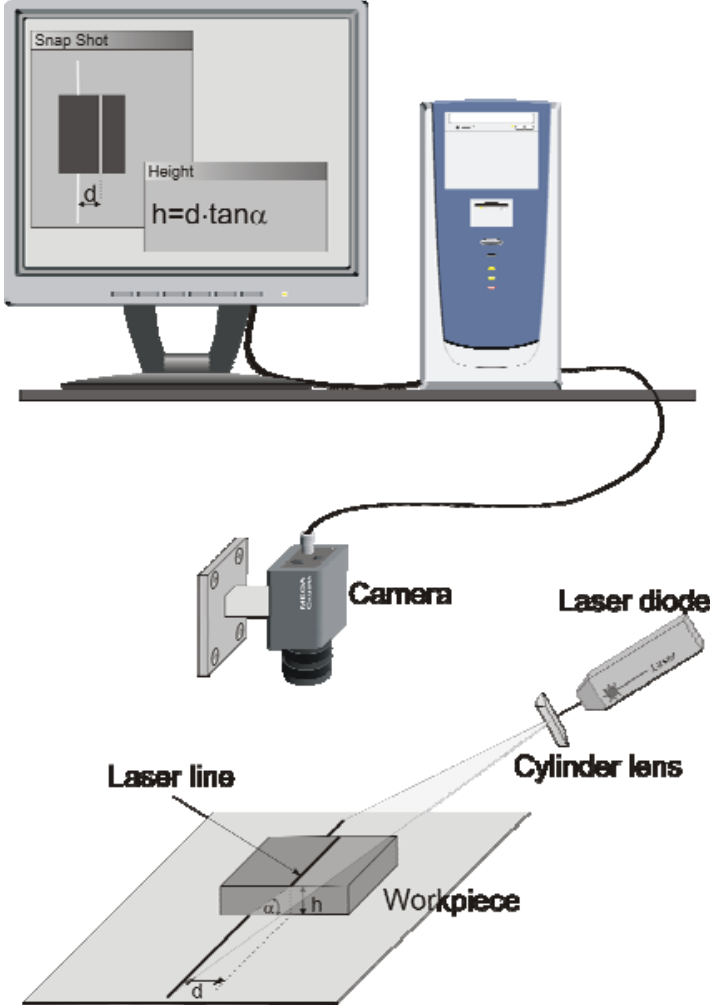

Figure 1. Working principle of a laser light sectioning sensor

In our laboratory setup we use a laser diode with a diffractive optical element that produces a set of seven parallel laser lines on the object. When trying to evaluate the shape of the lines we encountered problems with the speckle structures that were caused by the coherent light 
being scattered from a relatively rough surface. Fig. 2 shows a BGA component that was chosen for our experiments under normal (left) and under structured (right) illumination. The seven measurement lines are highlighted by the frame that is layed over the image. Additional less intensive laser lines are visible in the image. These are caused by higher order diffraction and will not be used for evaluation.

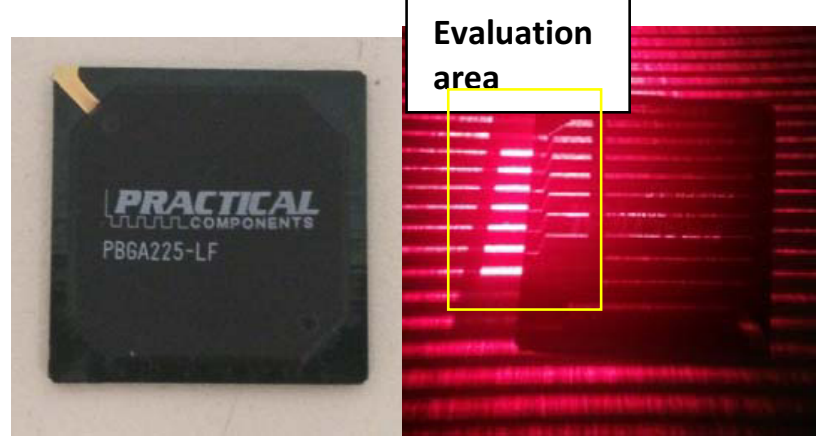

Figure 2. BGA component under normal (left) and stuctured (right) illumination

As can be seen from Fig. 3 the image of the laser lines is severely impaired by the speckle pattern. In the following we will describe a way to improve the image quality.

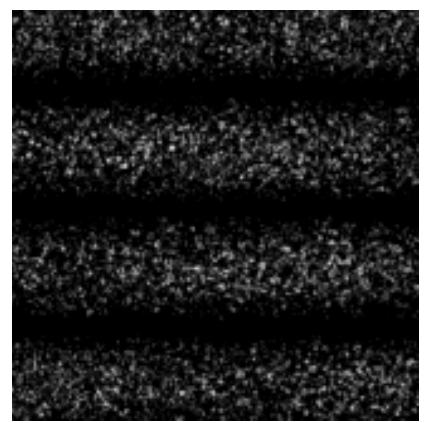

Figure 3. Magnified image of the laser lines with speckles

\section{Subjective Speckles}

When coherent light is scattered by a rough surface speckle patterns can be observed because various light rays are superimposed with statistically varying optical pathlengths, resulting in a stochastically varying local intensity. If a screen is directly illuminated by the scattered light we can measure statistically varying local intensity and call this "objective speckles". If the scattered light is imaged by a lens, so called "subjective speckles" arise. From a statistical analysis it can be derived that the average size $\bar{d}$ of a single speckle grain can be estimated [2]:

$$
\bar{d}=1.2 \frac{\lambda z_{O b j}}{D}
$$

with: $\lambda$ : wavelength of the light, $D$ : aperture diameter of the lens, $\mathrm{z}_{\mathrm{Obj}}$ : object distance
Applying the basic formulas of optical imaging

$$
\frac{1}{z_{\mathrm{Im}}}+\frac{1}{z_{O b j}}=\frac{1}{f}, \quad M=\frac{h_{\mathrm{Im}}}{h_{O b j}}=\frac{z_{\mathrm{Im}}}{z_{O b j}}, \quad F=\frac{f}{D}
$$

with: $\mathrm{z}_{\mathrm{Im}}$ : image distance, $\mathrm{h}_{\mathrm{Im}}$ : image height, $\mathrm{h}_{\mathrm{Obj}}$ : object height, $M$ : magnification, $F$ : aperture ratio or f/number

equation (1) can be written as [2]

$$
\bar{d}=1,2(1+M) \lambda F
$$

Apparently the structure of the pattern is governed by the aperture of the imaging lens, while the roughness parameters of the scattering surface can be shown to rule the intensity contrast. If an electronic image sensor with finite pixel size is used, the impact of the speckles can be reduced by using a lens with large aperture $D$, thus resulting in speckles smaller than the pixels of the camera. If several speckle grains are averaged by a pixel, the contrast of the pattern is reduced. Unfortunately the pixel size of modern electronic image sensors is so small that a small f-number of the lens would be required. The camera in our setup is a $\mu$ Eye UI-1480 SE with $2560 \mathrm{x}$ 1920 Pixels of $2.2 \mu \mathrm{m} \times 2.2 \mu \mathrm{m}$. With an object field of $40 \mathrm{~mm}$ the magnification is $M=0.14$. With the wavelength of the HeNe-laser being $\lambda=633 \mathrm{~nm}$ equation (3) reduces to $\bar{d}=0.87 \mu m F$. A significant reduction of speckle contrast due to averaging the intensity over the pixel area can only be expected, if the pixel size is larger than the average speckle size and this would require $F$ to be smaller than 2.5.

On the other hand, in the application of the light sectioning sensor we need a large depth of focus (DOF) of the optical imaging system and therefore the aperture ratio $F$ has to be set to a large number. Let $u$ be the maximum tolerated size of blur of an image point. The DOF can be estimated to be [3]

$$
D O F \approx u F \frac{1+M}{M^{2}}
$$

If we limit the tolerated image blur $u$ to the size of one pixel of our camera and set $F=2.5$ and $M=0.14$, the resulting DOF is only about $650 \mu \mathrm{m}$. In our application we need a DOF of at least $3 \mathrm{~mm}$ and therefore we have to step down the lens to $F=11$, giving us an average speckle size of about $9.5 \mu \mathrm{m}$ that is more than 4 pixels. Being aware that the speckle structure cannot be suppressed in the static images we decided to introduce a dynamic speckle reduction technique.

\section{Speckle contrast reduction dynamic averaging}

The speckle contrast $K$ is defined as:

$$
K=\frac{\sigma}{\langle I\rangle}
$$


with $\sigma$ : standard deviation of the intensity, $<\mathrm{I}>$ : average intensity

A relative lateral movement between the illuminated rough surface and the laser source reduces the contrast of the speckle pattern. In [4] the following formula is given:

$$
\frac{K}{K_{0}}=\sqrt{\frac{\tau_{c}}{2 T}\left[1-e^{\frac{-2 T}{\tau_{c}}}\right]}
$$

with $K_{0}$ : speckle contrast without movement, $T$ : image integration time, $\tau_{\mathrm{c}}$ : correlation time,

$$
\tau_{c}=A \frac{1}{v}
$$

with $v$ : velocity, $A$ : proportionality factor

We added a piezoelectric actuator to the illumination setup that imposed an oszillating movement of the laser lines along their axes. The higher the velocity, the lower is the correlation time $\tau_{\mathrm{c}}$. In order to reduce the speckle contrast, one has either to extend the integration time $T$ of the image sensor or to increase the velocity of the movement of the illuminating lightbeams. The time relation factor $2 T / \tau_{\mathrm{c}}$ can be used to control the resulting speckle contrast as is shown in Fig. 4.

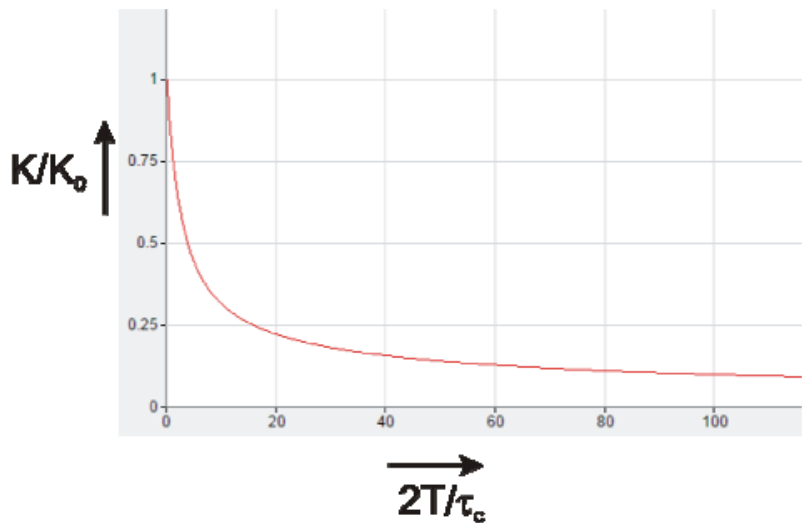

Figure 4. Relative speckle contrast as a function of time relation factor $2 T / \tau_{\mathrm{c}}$

\section{Experimental Results}

To verify our approach we experimentally determined the change of speckle contrast when varying the amplitude and the frequency of the oscillating movement. Fig. 5 shows the decrease of speckle contrast with increasing amplitude at a fixed oscillation frequency of $50 \mathrm{~Hz}$. Fig. 6 gives the corresponding relation for a fixed amplitude of $80 \mu \mathrm{m}$ and varying frequency. The integration time of the camera was set to $0.1587 \mathrm{~s}$ for all these measurements.

Using standard image processing algorithms we were able to reproducibly evaluate the line pattern with the reduced speckle contrast, while the original images without oscillating illumination posed severe problems. Fig. 7 shows a stepwise application of skeletonizing and line-thinning routines.

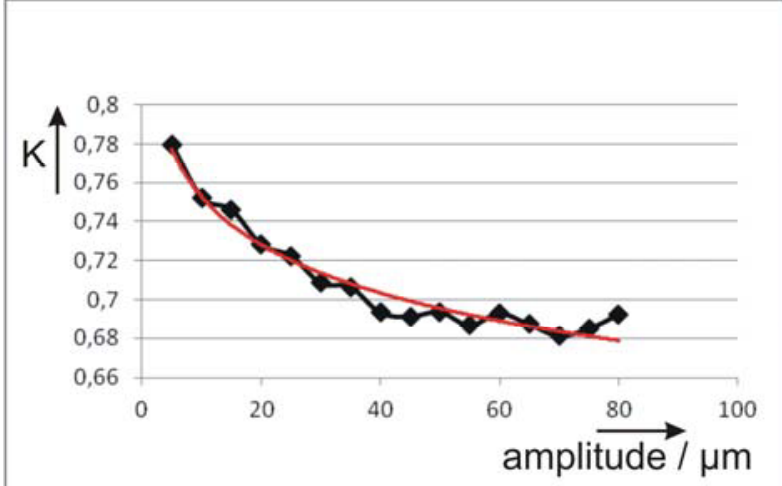

Figure 5. Speckle contrast as a function of oscillation amplitude

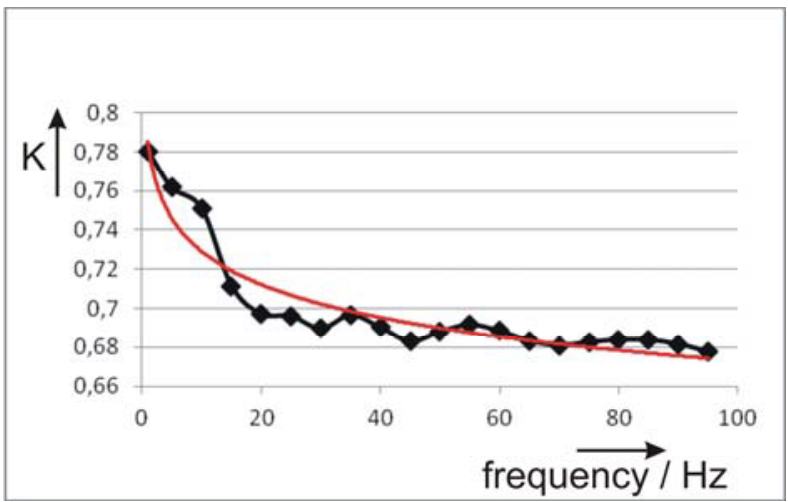

Figure 6. Speckle contrast as a function of oscillation frequency

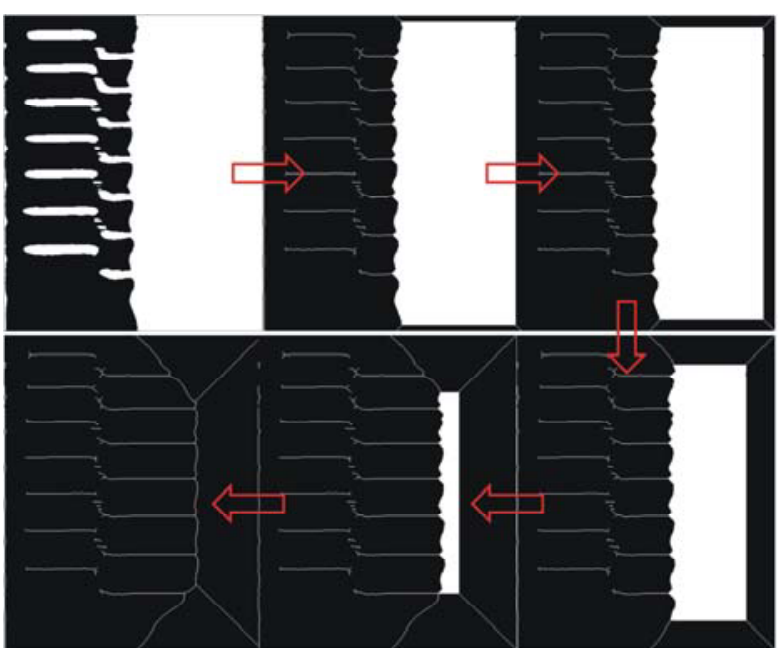

Figure 7. Demonstration of the evaluation of a line pattern with reduced speckle contrast.

\section{Conclusion}

The reduction of speckle-contrast caused by an oscillation of the line pattern has been used to increase the optical quality of the projected lines and to make automated evaluation by digital image processing robust. 


\section{References}

[1] G. Schmaltz: Technische Oberflächenkunde. Springer-Verlag (1936), ISBN: 978-3-642-51780-8

[2] A.E. Ennos: Speckle Interferometry. In: J.C. Dainty (Ed.): Laser Speckle and Related Phenomena. SpringerVerlag, (1975), ISBN 3-540-07498-8

[3] N.N.: Lexikon der Optik, part 2, p. 274, Spektrum Akademischer Verlag, (2003), ISBN 3-8274-1422-9

[4] J.D. Briers, Laser speckle contrast imaging for measuring blood flow, Optica Applicata, Vol. XXXVII, No. 1-2 (2007), 139-152 